Instituto Internacional de Investigación y Desarrollo Tecnológico Educativo INDTEC, C.A.

DOI: https://doi.org/10.29394/scientific.issn.2542-2987.2016.1.2.13.218-233

OAI-PMH: http://www.indteca.com/ojs/index.php/Revista Scientific/oai

\title{
Compromiso Social - Ético del Personal Docente desde el Paradigma de la Complejidad
}

\author{
Autoras: Marilú del Valle Parra Rojas \\ Universidad Nacional Experimental "Rafael María Baralt", UNERMB \\ mvlu27@gmail.com \\ Zulia, Venezuela \\ Sulenny Coromoto Mavarez Pernalete \\ Universidad Nacional Experimental "Rafael María Baralt", UNERMB \\ sulennymavarez@gmail.com \\ Zulia, Venezuela
}

Resumen

El objetivo de este estudio es comprometer social y éticamente al docente desde la perspectiva de la complejidad en las instituciones educativas. En principio las investigadoras recurren a la revisión bibliográfica enmarcada en las teorías socio-críticas que permean la acción del docente desde su compromiso social con autores como García y Martínez; asimismo cita a Morín y Rodrigo desde la perspectiva ética. Luego realizan el reconocimiento del escenario que les permite la descripción contextual y geográfica donde se encuentra la Escuela Básica Nacional "Héroe Nigale", de igual manera realizan el acercamiento al contexto donde se ejecutó la investigación y posteriormente se describen aspectos sociopolíticos del municipio Valmore Rodríguez; después se involucra a la comunidad seleccionada en el proceso de investigación, lo que permite recoger información a través de conversaciones y entrevistas con los informantes clave; generando de esta manera espacios para la sensibilización, reflexión y discusión con los actores sociales. Se inserta el paradigma cualitativo, bajo el método investigación-acción participativa IAP según Latorre (2008), aplicando la técnica de la entrevista a los docentes que laboran en la institución. Los resultados de esta investigación develan en los docentes el interés por superar las debilidades existentes en el proceso educativo, de igual manera fortalecer sus prácticas orientada hacia la transformación social, lo que implica ser responsables, equitativos, justos, enfocados a construir ciudadanos capaces de ser, aprehender, vivir y convivir con los otros en una misma sociedad.

Palabras clave: transformación; instituciones educativas; compromiso social; ética; docente. 


\title{
Social - Ethical Commitment of Teaching Personnel from the Paradigm of Complexity
}

\begin{abstract}
The aim of this study is ethically social compromise and teachers from the perspective of complexity in educational institutions. In principle, the researchers turn to the literature review framed in socio-critical theories that permeate teaching action from its social commitment with authors like Garcia and Martinez; also cites Rodrigo Morin and from an ethical perspective. Then perform the recognition of the scenario that allows them to contextual and geographical description where the "Hero Nigale" National Basic School, likewise made the approach to the context in which the investigation was carried out and subsequently socio-political aspects of the municipality Valmore Rodriguez described; then selected in the research process community is involved, which allows collecting information through conversations and interviews with key informants; thus generating awareness spaces for reflection and discussion with stakeholders. the qualitative paradigm is inserted under the participatory action research method IAP according Latorre (2008), applying the interview technique for teachers who work in the institution. The results of this research reveal in educational interest to overcome the weaknesses in the educational process, likewise strengthen oriented social transformation practices, which involves being responsible, equitable, fair, focused on building citizens capable of being, apprehend, live and coexist with others in the same society.
\end{abstract}

Keywords: transformation; educational institutions; social commitment; ethics; teacher. 


\section{Introducción}

La educación venezolana es una práctica social que presenta características especiales, comunicativa, dialógica, liberadora y transformadora, orientada con principios y justicias. Es por ello que el docente debe mantener un profundo compromiso ético con la educación que permita un aprendizaje esencial para la vida. En este sentido el proceso curricular venezolano del Ministerio del Poder Popular para la Educación” (2014); exige un compromiso- ético del docente que se alcance un esfuerzo continuo que se extienda en los espacios escolares, donde se accionen estrategias metodológicas para el aprender- aprender donde existan pertinencias, contextualizadas e innovadoras con desarrollo de habilidades que proporcionan el análisis reflexivo y crítico del contexto nacional para la construcción de una pedagogía propia.

En este sentido el docente debe ir más allá de transmisión de conocimiento quiere decir que sea transformador social a través de los proyectos educativos donde abarque una actuación constante, coherente con principios, que determine responsabilidades derivadas del compromiso ético.

Desde ese escenario, se observa que la Unidad Educativa Nacional Héroe Nigale presenta diferentes situaciones sobre el conocimiento práctico que implica el compromiso social- ético en el docente, es por ello que el objetivo de este estudio es comprometer social y éticamente al docente desde la perspectiva de la complejidad, donde se logre superar las debilidades existentes en el proceso educativo, así como también fortalecer sus prácticas educativas orientada hacia la transformación social para la educación de calidad del futuro como lo exige el Ministerio del Poder Popular para la Educación 


\section{Reconocimiento del Escenario donde se Ejecuta la Acción}

La investigación se desarrollara en el Municipio "Valmore Rodríguez "la misma se encuentra ubicado en la costa oriental del Lago de Maracaibo del Estado Zulia, Como se puede evidenciar la investigación se realizara en la parroquia la victoria perteneciente al mencionado municipio, específicamente en el sector la playa, el escenario donde se ejecuta la acciones es una la Unidad Educativa Nacional Héroe Nígale, la cual está ubicada en el sector la playa carretera los Teques limita al norte, con sector los Teques, al Sur río Misoa, al este con el sector curva el coquito, al Oeste con el lago de Maracaibo, atendiendo una matrícula de ciento ochenta y dos (182), distribuidos de la siguiente manera: 39 estudiantes de educación inicial, 70 en educación básica bolivariana, 73 de educación básica, media general y diversificada

\subsection{Descripción Contextual y Geográfica donde se encuentra la Institución}

La playa tiene aproximadamente 75 años de fundada (1930) Actualmente, cuenta con organizaciones internas como: asociaciones civiles, cooperativa y el consejo comunal conformado una población que supera los 466 habitantes.

Con respecto, al ámbito cultural, está comunidad no cuenta con instalaciones para desarrollar la cultura popular y la identidad de los habitantes, tomando para las actividades, la casa comunal, la escuela o en un terreno desolado que lo utilizan también para recrease con el popular juego bolas criollas.

En cuanto, al aspecto político, por ser este un sector rural desde el punto de vista de su posición geográfica, existen lineamientos políticos de diversas índoles, donde se destacan los partidos de gobierno, en los cuales gran porcentaje de la comunidad se encuentran inmersos por diferentes razones, unas de tipo económico, otra tendencia y otros por necesidad. 
Con relación a la actividad económica la desarrollan relacionando el cultivo con la cría de ganado destacándose con la producción de queso y leche; por otra parte, se desenvuelven con la pesca promoviendo al sector y al municipio Valmore Rodríguez una demanda alimentaria. En los que refiere a las familias cuentan con varios recursos económicos debido a la carencia de fuente de empleos formales, la mayoría trabaja en el comercio informal pescando y casas de familias.

\section{Percepción de las Investigadoras}

La educación Venezolana ha generado transformaciones con relación al sistema educativo donde el docente adquiera compromiso con las organizaciones educativas, cumpliendo un rol que responda a los planes de la nación referidos al ámbito educativo, es decir implica llevar la escuela hacia las comunidades brindando desde la formación académica apoyo y orientación en la resolución de problemas confrontados en la gestión escolar, lo que requiere una educación comprometida con las ideas democráticas, de libertad e igualdad como elemento que logre incorporar en la ciudadanía la idea de compromiso que humaniza al hombre.

En este sentido los nuevos lineamientos enmarcados en el documento "líneas estratégicas en el marco del proceso curricular venezolano del Ministerio del Poder Popular para la Educación" (2014); como ente rector en materia educativa, exige un compromiso- ético del docente que logre un esfuerzo sostenido que trascienda los espacios escolares, estrategias metodológicas de aprendizaje y enseñanza pertinentes, contextualizadas e innovadoras que desarrollen habilidades que facilitan el análisis reflexivo y crítico del contexto nacional e internacional y aporten a la construcción de una pedagogía propia.

De acuerdo a lo expuesto, esta investigación se sustente con los siguientes autores Méndez (2014) quien manifiesta que la calidad educativa 
es el proceso debe iniciarse a nivel gerencial del docente promoviendo una toma de conciencia sobre el rol del docente a través de la cual sea posible asimilar los nuevos paradigmas, no solo con la ciencia y tecnología, sino en función de la evolución cultural y la optimización del compromiso social que rodean la institución educativa. De tal manera que la educación pueda llenar un espacio significativo para mejorar la calidad de vida de las comunidades.

En otras palabras, en la Unidad Educativa Nacional Héroe Nigele se evidencia la ausencia de compromiso ético social de los docentes en este caso educativos, con respecto a las políticas educativas y planes de la nación.

\subsection{Acercamiento Realizado al Contexto}

Según, Mac Lean Alejandro (2010) Acercase a una comunidad de educando para ser investigado, es una acción que debe tomar en cuenta muchas situaciones, para consolidar una investigación el cual es indispensable recurrir a la comunicación escrita que establece el dialogo entre los autores donde se debe tener bastante cuidado y demasiado tacto, al establecer la comunicación escrita y oral con la población.

En este sentido, el acercamiento pretende la inserción de las investigadoras con la realidad para establecer relaciones de convivencia. Tal es el caso del grafico que se presenta a continuación que ocupa la caracterización de esta investigación como momento inicial para el diagnóstico; ya que el propósito es lograr el primer acercamiento. Y hacer relación con el personal, así como también dar a conocer el alcance del proyecto de investigación. 
Gráfico 1. Conversaciones.

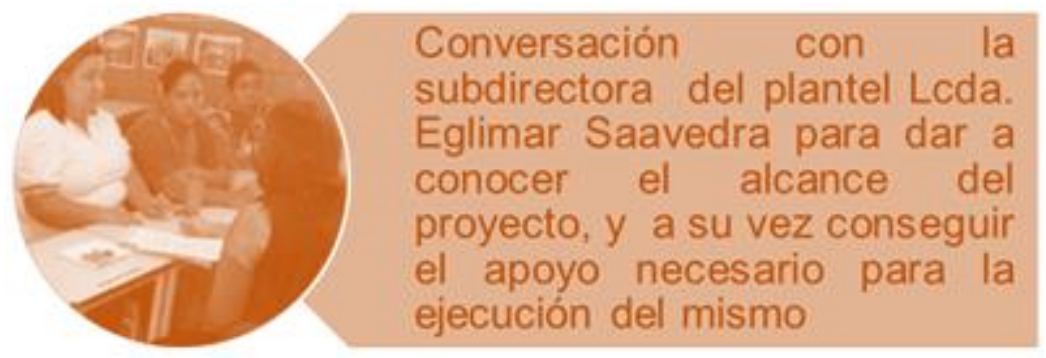

Conversación con los docentes del plantel, para dar a conocer el alcance del estudio, y establecer un clima de confianza.

Fuente: Parra 2015.

Al llevar a cabo el acercamiento para explorar el contexto real, se realizó la visita a la institución el día, 06-04-2015 donde se estableció una conversación pertinente con el representante del personal directivo Eglimar Saavedra, la cual tuvo como finalidad solicitar formalmente el permiso para desarrollar la investigación y conocer las características generales del plantel. De esta entrevista se logró la aprobación para el estudio y el suministro de información detallada del plantel en cuanto a la reseña histórica, organización, personal que labora y todo lo que respecta al contexto externo e interno del colectivo y la comunidad.

Una vez obtenida la información se programaron reuniones, así como, la entrega de invitaciones, publicación de carteles para dar a conocer a todo el personal que labora en la institución; además se establecieron acuerdos para realizar la siguiente acción de exponer el propósito de la investigación. 


\section{Fundamentación Teórica}

Ministerio del Poder Popular para la Educación (2015) propone que las Escuelas de Educación Primaria Bolivariana deben estar centrado bajo el compromiso del docente como parte de la convicción y transformaciones en materia educativa con la participación de todos los actores del proceso, considera que las Escuela, son transformadora de la sociedad en el cual se concrete e identifique con identidad nacional, práctica de participación, formación crítica, integración a la comunidad, el arraigo de la historia, costumbres, valores, que apunten a la construcción de una sociedad justa, fortaleciendo a las persona, comunidades para que sean protagonistas de la transformación del país.

En este mismo orden de ideas; García (2012), refiere que el compromiso es un elemento que se refiere a funcionar agregando valor a sus entornos comunitarios, la institución con respecto a los actores educativos, esto implica por parte de las instituciones en este caso la escuela debería reflexionar acerca de su trabajo, cual es el impacto que tiene en la comunidad, como contribuye al desarrollo endógeno de la misma y de la sociedad en general.

Es por ello, que docentes deben asumir compromiso con las organizaciones educativas donde cumplan y respondan a los planes de la nación referidos al ámbito educativo, es decir implica llevar la escuela hacia las comunidades brindando desde la formación académica apoyo y orientación en la resolución de problemas confrontados en la gestión escolar, lo que requiere mayor compromiso para lograr equipos de docentes investigadores de su hacer educativo en conjunto con la comunidad e institución en general.

Tomando en cuenta lo anterior es necesario mencionar en este artículo que la ética de docente es importante para la transformación y la calidad educativa en la instituciones escolares por ello la conducta del ser humano es primordial para la toma de conciencia y el desarrollo integral de cada individuo 
para ello se toma en cuenta a Morín (2010) quien refiere que la ética del género humano orienta a la educación a contribuir en la toma de conciencia del cuidado del país, así como las personas que circundan en la comunidad, está conciencia se debe concebir en la voluntad de producir verdaderos cambios en la manera de pensar y actuar.

Así mismo agrega que la ética no se enseña con clases de moral o valores, sino que cada persona debe preocuparse por su desarrollo integral como, individuo, perteneciente de una comunidad o sociedad donde se exige un verdadero compromiso social.

Quiere decir que la ética del docente conlleva cambios y transformación en todos los elementos que implica el sistema educativo, donde el docente, debe comprometerse y trascender más allá del conocimiento, ser quien conlleve a través de proyecto educativo la participación de todas y todos los actores educativos,

De igual forma Rodrigo (2011) refiere que desafío ético del docente está en correspondencia con la sociedad educativa donde se reproducirán y desarrollarán conocimientos, competencias, valores necesarios para una satisfactoria vida económica, social, personal. Quiere decir que la ética del docente nos permite ver no solamente su función sino la integralidad social de todos los actores del hecho educativo

Para Cortina (2013) la ética es considerado con la moral que consistente en la realización de un bien subjetivo, donde la obtención de un bien deseado reside en el cumplimiento de un objetivo independiente del deseo del sujeto, donde el objetivo pueda consistir en la perfección del individuo o en la perfección progresiva de la sociedad. En este orden de ideas la ética es un paradigma empírico que mueve al hombre, al perfeccionamiento y la plenitud, donde radica el bien de todo hombre. De igual forma afirma que la ética determina la base de la moral, ya que considera que se debe tener claro el significado de "moral" como capacidad para enfrentar la vida frente a 
"desmoralización", para lo cual hay que tener "altura moral", algo que la filósofa sostiene que no puede venir del exterior ni que se trata de deberes que hay que imponer, sino que tiene que nacer del hombre mismo hasta alcanzar su plenitud.

Con base a lo anterior se enfatiza sobre la complejidad del compromiso social-ético del personal docente según Hernández (2011) parte de la filosofía refiere que la ética y el compromiso se obtiene a través de la conducta humana, es decir, las reglas, principios que todo ser humano debe ajustarse a su actuación. De ahí las acciones de las personas con relación al comportamiento y objetivos que se desean alcanzar.

En virtud a lo planteado el autor mencionado plantea que el docente en la actual sociedad compleja es quien a través de las actividades sociales proporciona el fortalecimiento del entorno comunitario con la finalidad de mantener un bienestar en el desarrollo y el progreso necesario para el entorno o la sociedad que circunda en la institución. De igual forma el docente es quien de manera responsable se compromete con el logro de los objetivos que la institución educativa tiene, tomando en cuenta las políticas educativas,

Desde esta perspectiva de la complejidad de la educación debe ser diferente si cada uno asume no sólo un código de ética sino las normas que colectivamente ayuden a los ciudadanos a ser mejores hombres cada día y a identificar las relaciones entre humanos como las más convenientes pero no hacia una moral sino hacia la mejor manera de ser felices; es decir, asumir el compromiso.

A esto se le acuña lo que plantea Flores (2015) que la complejidad del hombre, como ser social se hace en la medida en que es educado, quiere decir que el proceso de aprendizaje humano desde el punto de vista de su incorporación al mundo, por su naturaleza social se adapta a la vida organizada con otros seres humanos, ya que la mirada de la educación 
propone la construcción de un "hombre nuevo", con sentido de compromiso con el mundo y la sociedad.

Pues quiere decir entonces que el docente es quien transmite la enseña al entorno social conllevando a la construir un hombre nuevo desde el compromiso ético para la vida futura, siguiendo sus necesidades, transformando de cada individuo desde la manera de pensar hasta la conducta adquirida.

\section{Metodología y Resultados}

Para el desarrollo de este trabajo se seleccionó la investigación-acción educativa la cual tiene como propósito según Latorre (2008); dirigir al docente a la reflexione sobre su práctica educativa, de forma que repercuta, tanto sobre la calidad del aprendizaje como la propia enseñanza, es decir, hace que el docente actúe como investigador e investigado, simultáneamente.

El modelo propuesto de acuerdo a Serrano (2010); está relacionado en ejecutar acción transformadora colectivamente con la participación de los involucrados en la problemática, quiere decir se comienza con una planeación de una práctica-acción transformadora donde actúen los docentes, en una continua reflexión sobre la praxis diaria

La técnica utiliza poyado en Serrano (2010) es la observación y la entrevista, esto significa que le investigador(a) se convierte en un ente motivador del proceso y protagonista del fenómeno de estudio.

De acuerdo a Ander-Egg (2011), La observación participante consiste, en esencia, en la observación del contexto desde la perspectiva de la propia investigadora o investigador de una forma no encubierta y no estructurada. Se alarga en el tiempo sin la utilización de matrices ni códigos estructurados previamente, sino que se hace a partir de la inmersión en el contexto. Cabe mencionar, que la investigadora es miembro del grupo de estudio, lo cual facilita la observación, dado que ha socializado con ellos conoce su 
comportamiento, actuación, no necesita hacer ninguna negociación para tener acceso al escenario real de los hechos.

De igual manera Galindo (2013), expone que la entrevista cualitativa se define como aquélla no directiva, abierta, no estructurada, ni estandarizada, la cual sigue un modelo conversacional, superando la perspectiva de un intercambio formal de preguntas y respuestas en la medida que trata de emular un diálogo entre iguales. En cuanto a las entrevistas a realizar para el desarrollo investigativo actual, se harán de manera informal a fin de lograr la confianza plena de la persona entrevistada.

Asimismo, para la recolección de la información y obtención de los resultados se utilizó el instrumento registro de campo Dávila (2011). El registro de campo es un instrumento utilizado por el investigador para registrar aquellos hechos susceptibles de ser interpretados, en este sentido el diario de campo es una herramienta que permite sistematizar las experiencias para luego analizar los resultados.

\section{Análisis de los Resultados}

Sistematización del resultado por medio de las acciones realizadas se pudo determinar que el compromiso social - ético de los actores del proceso educativo están en que deben asumir el mismo con las organizaciones educativas y comienza cuando los docentes cumplen con su rol, para responder a los planes conferidos por políticas, planes, programas y proyectos referidos en el plan de la nación consagrados en la LOE, que son ineludible a la formación del ser humano y emanados por el sistema educativo, es decir implica llevar la escuela hacia una cultura de participación, para transformar las realidades de las comunidades brindando la formación académica el apoyo y la orientación para el mejoramiento de problemas que confrontan durante la gestión escolar. 


\section{Conclusiones}

Considerando que el objetivo del estudio es comprometer social y éticamente al docente desde la perspectiva de la complejidad en las instituciones educativas; es importante resaltar lo siguiente: En cuanto al compromiso social la reflexión profunda es que el docente debe asumir el rol de una persona analítico, reflexivo, para transformar del modelo educativo de la educación del futuro teniendo en cuenta que el ser humano debe ser integral, de igual forma es necesario incluir la transdisciplinariedad en los planes de estudios si se desea para formar personas competentes, cooperativas, criticas, democráticas, con la finalidad de la comprensión del hombre en la interacción con el mundo.

Así mismo tomar la integración de saberes, métodos, valores y principios, brindando la oportunidad al ser humano de seguir estudiando a lo largo de su vida, aprender de diversas formas y desde diferentes fuentes (teóricas, tecnológicas, otras). De igual forma, en relación a la ética y a la complejidad que se desarrolla en las instituciones educativas es la actitud que se adopta desde el ser, significa las capacidades de autoaprendizaje, para adaptarse a un tiempo de cambios permanentes, y así comprender la complejidad del mundo donde le toca vivir, traspasando las barreras, fronteras de cultura, idiomas, sociedad, donde se rompan paradigmas, dejando de lado las ideas antiguas que no han dado resultados favorables, para dar paso a cambios significativos

\section{Referencias}

Cortina (2013). “¿Para qué sirve realmente la ética?”. Barcelona: Universidad de Valencia.

Dávila, O. (2011). "Ética y educación para el nuevo siglo". En Revista Educación, Año/vol. 29, No. 1. Universidad de Costa Rica. Ciudad Universitaria Rodrigo Facio, Costa Rica. Publicación de Hernández. 
Ley Orgánica de Educación (2009). Gaceta Oficial № 5.929 Extraordinario del 15 de agosto. República Bolivariana de Venezuela: La Asamblea Nacional.

Ministerio del Poder Popular para la Educación (2014). Líneas estratégicas en el marco del proceso curricular venezolano. Venezuela.

Morín, E. (2010). Los siete Saberes Necesarios para la Educación del Futuro. Publicado por Vallejos, en octubre de 1999. UNESCO.

Rodrigo (2011). Conferencia: "La profesionalidad moral: valores éticos en la formación universitaria", en Rodrigo López Zavala (coord.), Huellas de la profesionalidad. Ética profesional en la formación universitaria, México, UAS, Plaza y Valdés, pp. 11-37.

Latorre, A. (2008). La investigación acción. Conocer y cambiar la práctica educativa. Barcelona: Editorial Graó. Recuperado de:

http://revistas.uis.edu.co/index.php/revistadocencia/article/download/2 $\underline{451 / 2778 /}$

Serrano (2010). Desafíos de la Investigación Cualitativa. Catedrática de Pedagogía Social Universidad Nacional de Educación a Distancia (UNED). Chile.

Ander-Egg, E. (2011). "Repensando la investigación acción participativa". Cuarta edición. Grupo editorial Lumen. Humanitas.

García (2012). Un compromiso de los actores socioeducativos para cambiar las escuelas. Congreso Internacional de Transformación Educativa.

Méndez (2014). Calidad Educativa. Conferencia en el marco del proceso curricular venezolano del Ministerio del Poder Popular para la Educación. MPPE.

Flores, J. (2015). Complejidad y educación. Editorial Universidad Don Bosco. [Documento en línea]. Recuperado de: http://hdl.handle.net/10972/2048 
Nacida en Ciudad Ojeda estado Zulia Venezuela,

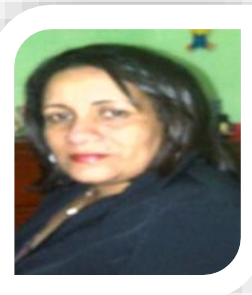
cursó estudios de pregrado en la Universidad Nacional Experimental Rafael María Baralt obteniendo título de Lcda. Educación Integral, posteriormente realizó estudios de postgrado en Evaluación por la Universidad Valle del Momboy, estado Trujillo, es Cursante del Doctorado de Educación de la Universidad Nacional Experimental Rafael María Baralt, estado Zulia, y actualmente se encuentra en espera del título como Magister en Gerencia Educativa de la Nororiental Gran Mariscal de Ayacucho. En la actualidad se desempaña como Subdirectora de la Unidad Educativa Nacional Bolivariana Daniel Mavarez, con 13 años al servicio del Ministerio del Poder Popular para la Educación. 


\section{Sulenny Coromoto Mavarez Pernalete}

e-mail: sulennymavarez2@gmail.com

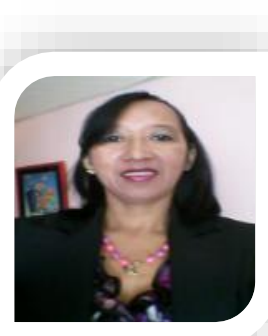

Nacida en Ciudad Ojeda, estado Zulia, Venezuela, cursó estudios de pregrado en la Universidad Nacional Experimental "Rafael María Baralt" (UNERMB), obteniendo el título de Lcda. Educación Mención Integral, además cursó estudios de pregrado en la Universidad del Zulia (LUZ), obteniendo el título de Lcda. Educación Menciones Matemática y Física, posteriormente realizó estudios de postgrado en Didáctica de Las Matemáticas en la Universidad Valle del Momboy (UVM), estado Trujillo, es Cursante del Doctorado en Educación en la Universidad Nacional Experimental "Rafael María Baralt" (UNERMB), estado Zulia. En la actualidad se desempaña como Docente de aula por horas en la Unidad Educativa Nacional "Campo Lara", con 15 años al servicio del Ministerio del Poder Popular para la Educación.

El contenido de este manuscrito se difunde bajo una Licencia de Creative Commons ReconocimientoNoComercial-Compartirlgual 4.0 Internacional 